Supporting Information

\title{
A Simple and Scalable Protocol for Producing Hydrophobic Polymer Brushes Beyond Wafer-Scale Dimensions Toward Real-Life Applications
}

\author{
Tomoya Sato, ${ }^{1}$ Gary J. Dunderdale, ${ }^{2}$ Atsushi Hozumi ${ }^{1 *}$ \\ ${ }^{1}$ National Institute of Advanced Industrial Science and Technology (AIST), \\ 2266-98, Anagahora, Shimo-shidami, Moriyama, Nagoya, Japan, 463-8560 \\ ${ }^{2}$ Department of Biological and Chemical Engineering, University of Sheffield, \\ Sheffield, UK S1 3JD
}

*E-mail: a.hozumi@aist.go.jp

\section{Table of Contents}

1) Table S1 Dry thicknesses, number average molecular weight $\left(M_{\mathrm{n}}\right)$, dispersity $(\bigoplus)$, grafting density $(\sigma)$, water contact angles (CAs), and root-mean-square roughness $\left(R_{\mathrm{rms}}\right)$ values of poly (methyl methacrylate) (PMMA) brushes grafted from silicon ( $\mathrm{Si}$ ) substrates covered with surface initiator layer (SIL) at different ethanol/water mixing ratios

2) Table S2 Dry thicknesses, water CAs, and $R_{\text {rms }}$ values of three different hydrophobic polymer brushes grafted from SIL-formed Si substrates by water-accelerated "paint-on" SI-ATRP at different reaction time

3) Table S3 Dry thicknesses, water CAs, and $R_{\text {rms }}$ values of a wide variety of hydrophobic polymer brushes grafted by water-accelerated "paint-on” SI-ATRP from SIL-formed various inorganic/organic substrates

4) Figure S1. Schematic illustration of setup for water-accelerated "paint-on" SI-ATRP demonstrated on SIL-grafted large-scale substrates. 
5) Figure S2. Color changes of reaction solutions for water-accelerated "paint-on" SI-ATRP of methyl methacrylate (MMA) during ( $\sim 1$ min, after activation) and after (completely deactivated) the reaction. 6) Figure S3. Typical optical microscope $\left(\mathrm{OM}\right.$, viewing area of $\left.300 \times 300 \mu \mathrm{m}^{2}\right)$ images of PMMA brush-grafted Si substrates prepared by water-accelerated "paint-on" SI-ATRP at different ethanol/water mixing ratio.

7) Figure S4. Typical atomic force microscope (AFM, viewing area of $3 \times 3 \mu \mathrm{m}^{2}$ ) images of PMMA brush-grafted Si substrates prepared by water-accelerated "paint-on" SI-ATRP at different ethanol/water mixing ratio.

8) Table S4 Dry thicknesses, water CAs, and $R_{\mathrm{rms}}$ values of the resulting PMMA brushes grafted from SIL-formed Si substrates by water-accelerated "paint-on” SI-ATRP using stored solutions for 24 or $240 \mathrm{~h}$

9) Table S5 Dry thicknesses of the resulting PMMA brushes grafted from SIL-formed Si substrates by water-accelerated "paint-on" SI-ATRP at various monomer concentrations

10) Figure S5. Increases in thicknesses and thicknesses ratio of a wide variety of hydrophobic polymer brushes prepared by water-accelerated "paint-on" SI-ATRP comparing to "paint-on" SI-ATRP without addition of water.

11) Figure S6. Appearance and distributions of water CAs of the poly $(1 H, 1 H, 2 H, 2 H$ heptadecafluorodecyl acrylate) (PHDFDA) brush layer formed on large-scale glass substrate.

12) Table S6 Static and dynamic CAs of five probe liquids on PHDFDA brush-grafted Si substrates ( $\theta_{\mathrm{A}}$ : advancing $\mathrm{CA}, \theta_{\mathrm{R}}$ : receding $\mathrm{CA}, \Delta \theta$ : CA hysteresis, and $\theta_{\mathrm{T}}$ : sliding/tilt angle)

13) Movie S1. Motion of water droplet placed on the inclined PHDFDA brush-grafted glass slide (5 $\mathrm{cm} \times 8 \mathrm{~cm})$.

14) Movie S2. Motion of $n$-hexane droplet placed on the inclined PHDFDA brush-grafted glass slide $(5 \mathrm{~cm} \times 8 \mathrm{~cm})$.

15) Movie S3. Continuous water jet flow on PHDFDA brush-grafted glass slide $(5 \mathrm{~cm} \times 8 \mathrm{~cm})$.

16) Movie S4. Continuous oil ( $n$-hexane) jet flow on PHDFDA brush-grafted glass slide $(5 \mathrm{~cm} \times 8$ $\mathrm{cm})$.

\section{Number of pages: 14}

Number of Figures: 6

Number of Tables: 6 
Table S1 Dry thicknesses, number average molecular weight $\left(M_{\mathrm{n}}\right)$, dispersity $(\nexists)$, grafting density $(\sigma)$, water contact angles (CAs), and root-mean-square roughness $\left(R_{\text {rms }}\right)$ values of poly (methyl methacrylate) (PMMA) brushes grafted from silicon (Si) substrates covered with surface initiator layer (SIL) at different ethanol/water mixing ratios

\begin{tabular}{|c|c|c|c|c|c|c|c|c|c|c|c|c|}
\hline Entry & Substrate & Monomer & $\begin{array}{c}{[\text { monomer }]} \\
/\left[\mathrm{CuCl}_{2}\right] \\
/[\mathrm{PMDETA}] \\
/[\mathrm{AA}]\end{array}$ & $\begin{array}{c}\text { Ethanol } \\
\text { (vol\% in } \\
\text { solvent) }\end{array}$ & $\begin{array}{c}\text { Water } \\
\text { (vol\% in } \\
\text { solvent) }\end{array}$ & $\begin{array}{c}\text { Reaction } \\
\text { time } \\
\text { (min) }\end{array}$ & $\begin{array}{l}\text { Thickness } \\
\text { (nm) }\end{array}$ & $M_{\mathrm{n}}$ & D & $\begin{array}{c}\sigma \\
(\text { chains } \\
\left./ \mathrm{nm}^{2}\right)^{\mathrm{a}}\end{array}$ & $\begin{array}{l}\text { Water } \\
\text { CA } \\
\left(^{\circ}\right)\end{array}$ & $\begin{array}{l}R_{\mathrm{rms}} \\
\text { from } \\
\text { AFM } \\
(\mathrm{nm}) \\
\end{array}$ \\
\hline 1 & & & & 100 & 0 & 15 & 16.0 & 27000 & 1.30 & 0.42 & 79 & 0.75 \\
\hline 2 & & & & 99 & 1 & 15 & 30.9 & 24400 & 1.19 & 0.90 & 79 & 0.77 \\
\hline 3 & & & & 98 & 2 & 15 & 43.6 & 39800 & 1.18 & 0.77 & 80 & 0.71 \\
\hline 4 & & & 1.5 & 83 & 17 & 15 & 39.3 & 55200 & 1.02 & 0.51 & 78 & 1.4 \\
\hline 5 & $\mathrm{Si}$ & MMA & $\begin{array}{l}/ 4 \times 10^{-4} \\
/ 1 \times 10^{-3}\end{array}$ & 67 & 33 & 15 & 38.2 & 49400 & 1.55 & 0.54 & 80 & 1.3 \\
\hline 6 & & & $/ 1 \times 10^{-3}$ & 50 & 50 & 15 & 32.5 & 37300 & 1.21 & 0.72 & 78 & 2.5 \\
\hline 7 & & & & 33 & 67 & 15 & 13.2 & 12300 & 1.30 & 0.76 & 84 & 0.24 \\
\hline 8 & & & & 17 & 83 & 15 & 9.5 & $-\mathrm{b}$ & $-b$ & - & 86 & 0.26 \\
\hline 9 & & & & 0 & 100 & 15 & 5.1 & $-\mathrm{b}$ & $-\mathrm{b}$ & - & 85 & 0.23 \\
\hline
\end{tabular}

${ }^{a}$ roughly calculated with thickness and $M_{\mathrm{n}}$ according to eq. $\mathrm{S} 1,{ }^{\mathrm{b}}$ not detectable because of extremely low concentration of polymers.

(eq. S1) Grafting density $(\sigma)=d \times L_{\mathrm{d}} \times N_{\mathrm{A}} \times 10^{-21} / M_{\mathrm{n}}$

where, $h, \rho$ and $N_{\mathrm{A}}$ are the thickness of PMMA brushes, density of bulk PMMA at $293 \mathrm{~K}(1.18$ $\left.\mathrm{g} / \mathrm{cm}^{3}\right)$, and Avogadro's number $\left(6.02 \times 10^{23}\right)$, respectively [1].

\section{De-grafting of PMMA Brushes from Si Substrates}

De-grafting of PMMA brushes were demonstrated according to a previously reported process using tetra $n$-butylammonium fluoride (TBAF) as a cleaving agent [2]. Each PMMA-grafted Si wafer $(1 \mathrm{~cm} \times 2 \mathrm{~cm})$ was put in a glass jar containing a $25 \mathrm{~mL}$ solution of $0.04 \mathrm{M}$ TBAF in THF and incubated at $55^{\circ} \mathrm{C}$ for $24 \mathrm{~h}$. The solution was then collected and concentrated with nitrogen blow. The resulting solutions (about $1 \mathrm{~mL}$ ) were filtrated 
through $0.22 \mu \mathrm{m}$ polytetrafluoroethylene filters and passed through the gel permeation chromatography (GPC). Number average molecular weight $\left(M_{\mathrm{n}}\right)$, weight average molecular weight $\left(M_{\mathrm{w}}\right)$, and dispersity $\left(\oslash, M_{\mathrm{w}} / M_{\mathrm{n}}\right)$ were estimated from PMMA standards. (Note that because quantities of the de-grafted PMMAs were extremely low, signals of some samples could not be detected by the GPC system used. Hence, the values shown in Table S1 were just references.)

\section{Surface-initiated (SI)-ARGET-ATRP of Various Hydrophobic Monomers in}

\section{Ethanol}

Hydrophobic polymer brushes containing various functional groups were grafted through SI-ARGET-ATRP process on SIL-formed Si substrates. A proper amount of monomer (concentrations were set at $50 \mathrm{vol} \%$ for liquid monomers and $25 \mathrm{vol} \%$ for solid monomers), $\mathrm{CuCl}_{2}$, PMEDTA, and ethanol were added to a glass vial with a piece of surface initiator layer (SIL)-formed Si substrate $(2 \mathrm{~cm} \times 1 \mathrm{~cm})$, and mixed (typical ratios were listed in Table S2). Freshly prepared ethanolic solution of AA (10 mg/mL) was then added to the reaction solutions and stirred for about $5 \mathrm{sec}$ to activate the catalyst. Polymerizations were allowed to proceed at room temperature under ARGET conditions without stirring. After $15 \mathrm{~min}$, the substrates were removed from the solution. They were then extensively rinsed with some of the good solvents including toluene, AK-225 (for perfluorinated polymer brushes), ethanol and Mill-Q water repeatedly to remove excess monomer, and were dried with a $\mathrm{N}_{2}$ blowing at room temperature. 
Table S2 Dry thicknesses, water CAs, and $R_{\text {rms }}$ values of three different hydrophobic polymer brushes grafted from SIL-formed Si substrates by water-accelerated "paint-on" SI-ATRP at different reaction time

\begin{tabular}{|c|c|c|c|c|c|c|c|c|c|}
\hline Entry & Substrate & Monomer & $\begin{array}{c}{[\text { monomer }] /\left[\mathrm{CuCl}_{2}\right]} \\
\text { /[PMDETA]/[AA] }\end{array}$ & $\begin{array}{c}\text { Ethanol } \\
\text { (vol\% in } \\
\text { solvent) }\end{array}$ & $\begin{array}{c}\text { Water } \\
\text { (vol\% in } \\
\text { solvent) }\end{array}$ & $\begin{array}{l}\text { Reaction } \\
\text { time } \\
\text { (min) }\end{array}$ & $\begin{array}{l}\text { Thickness } \\
\text { (nm) }\end{array}$ & $\begin{array}{l}\text { Water } \\
\text { CA } \\
\left(^{\circ}\right)\end{array}$ & $\begin{array}{c}R_{\mathrm{rms}} \\
\text { from } \\
\text { AFM } \\
(\mathrm{nm})\end{array}$ \\
\hline 10 & \multirow{7}{*}{$\mathrm{Si}$} & \multirow{7}{*}{ MMA } & \multirow{7}{*}{$\begin{array}{c}1.5 / 4 \times 10^{-4} \\
/ 1 \times 10^{-3} / 1 \times 10^{-3}\end{array}$} & \multirow{7}{*}{98} & \multirow{7}{*}{2} & 5 & 26.3 & 79 & 0.40 \\
\hline 11 & & & & & & 10 & 33.4 & 78 & 0.62 \\
\hline 12 & & & & & & 15 & 42.8 & 79 & 0.68 \\
\hline 13 & & & & & & 30 & 44.3 & 74 & 0.28 \\
\hline 14 & & & & & & 60 & 51.4 & 78 & 0.40 \\
\hline 15 & & & & & & 120 & 60.1 & 78 & 0.69 \\
\hline 16 & & & & & & 360 & 74.3 & 76 & 0.39 \\
\hline 17 & \multirow{7}{*}{$\mathrm{Si}$} & \multirow{7}{*}{ MA } & \multirow{7}{*}{$\begin{array}{c}1.9 / 4 \times 10^{-4} \\
/ 1 \times 10^{-4} / 1 \times 10^{-3}\end{array}$} & \multirow{7}{*}{98} & \multirow{7}{*}{2} & 5 & 21.7 & 90 & 0.31 \\
\hline 18 & & & & & & 10 & 25.3 & 89 & 0.34 \\
\hline 19 & & & & & & 15 & 30.4 & 83 & 0.85 \\
\hline 20 & & & & & & 30 & 57.9 & 84 & 0.83 \\
\hline 21 & & & & & & 60 & 67.1 & 88 & 0.28 \\
\hline 22 & & & & & & 120 & 77.0 & 86 & 0.44 \\
\hline 23 & & & & & & 360 & 84.2 & 82 & 0.26 \\
\hline 24 & \multirow{7}{*}{$\mathrm{Si}$} & \multirow{7}{*}{ St } & \multirow{7}{*}{$\begin{array}{c}1.4 / 4 \times 10^{-4} \\
/ 1 \times 10^{-4} / 1 \times 10^{-3}\end{array}$} & \multirow{7}{*}{98} & \multirow{7}{*}{2} & 5 & 26.2 & 84 & 0.50 \\
\hline 25 & & & & & & 10 & 31.0 & 90 & 0.46 \\
\hline 26 & & & & & & 15 & 47.7 & 94 & 0.42 \\
\hline 27 & & & & & & 30 & 58.1 & 94 & 0.77 \\
\hline 28 & & & & & & 60 & 61.0 & 91 & 0.48 \\
\hline 29 & & & & & & 120 & 62.6 & 89 & 0.37 \\
\hline 30 & & & & & & 360 & 82.0 & 91 & 0.44 \\
\hline
\end{tabular}


Table S3 Dry thicknesses, water CAs, and $R_{\text {rms }}$ values of a wide variety of hydrophobic polymer brushes grafted by water-accelerated "paint-on" SI-ATRP from SIL-formed various inorganic/organic substrates

\begin{tabular}{|c|c|c|c|c|c|c|c|c|c|}
\hline Entry & Substrate & Monomer & $\begin{array}{c}{[\text { monomer }] /\left[\mathrm{CuCl}_{2}\right]} \\
/[\mathrm{PMDETA}] /[\mathrm{AA}]\end{array}$ & $\begin{array}{l}\text { Ethanol } \\
\text { (vol\% in } \\
\text { solvent) }\end{array}$ & $\begin{array}{l}\text { Water } \\
\text { (vol\% in } \\
\text { solvent) }\end{array}$ & $\begin{array}{l}\text { Reaction time } \\
\quad(\min )\end{array}$ & $\begin{array}{l}\text { Thickness } \\
\text { (nm) }\end{array}$ & $\begin{array}{c}\text { Water } \\
\text { CA } \\
\left(^{\circ}\right) \\
\end{array}$ & $\begin{array}{l}R_{\mathrm{rms}} \\
\text { from } \\
\text { AFM } \\
(\mathrm{nm}) \\
\end{array}$ \\
\hline 31 & \multirow{12}{*}{$\mathrm{Si}$} & MMA & $\begin{array}{c}1.5 / 4 \times 10^{-4} \\
/ 1 \times 10^{-3} / 1 \times 10^{-3}\end{array}$ & 98 & 2 & 15 & 48.0 & 79 & 0.52 \\
\hline 32 & & MA & $\begin{array}{c}1.9 / 4 \times 10^{-4} \\
/ 1 \times 10^{-3} / 1 \times 10^{-3}\end{array}$ & 98 & 2 & 15 & 48.5 & 81 & 1.02 \\
\hline 33 & & BMA & $\begin{array}{c}1.1 / 4 \times 10^{-4} \\
/ 1 \times 10^{-3} / 1 \times 10^{-3}\end{array}$ & 98 & 2 & 15 & 71.0 & 89 & 0.42 \\
\hline 34 & & HMA & $\begin{array}{c}0.95 / 4 \times 10^{-4} \\
/ 1 \times 10^{-3} / 1 \times 10^{-3}\end{array}$ & 98 & 2 & 15 & 65.2 & 93 & 0.39 \\
\hline 35 & & St & $\begin{array}{c}1.4 / 4 \times 10^{-4} \\
/ 1 \times 10^{-3} / 1 \times 10^{-3}\end{array}$ & 98 & 2 & 15 & 65.3 & 94 & 0.71 \\
\hline 36 & & BA & $\begin{array}{c}1.2 / 4 \times 10^{-4} \\
/ 1 \times 10^{-3} / 1 \times 10^{-3}\end{array}$ & 98 & 2 & 15 & 72.3 & 97 & 0.49 \\
\hline 37 & & TFEMA & $\begin{array}{c}1.2 / 4 \times 10^{-4} \\
/ 1 \times 10^{-3} / 1 \times 10^{-3}\end{array}$ & 98 & 2 & 15 & 19.2 & 97 & 0.53 \\
\hline 38 & & HA & $\begin{array}{c}0.95 / 4 \times 10^{-4} \\
/ 1 \times 10^{-3} / 1 \times 10^{-3}\end{array}$ & 98 & 2 & 15 & 78.7 & 105 & 0.29 \\
\hline 39 & & EHA & $\begin{array}{c}0.81 / 4 \times 10^{-4} \\
/ 1 \times 10^{-3} / 1 \times 10^{-3}\end{array}$ & 98 & 2 & 15 & 118.2 & 105 & 0.40 \\
\hline 40 & & StA & $\begin{array}{c}0.13 / 4 \times 10^{-4} \\
/ 1 \times 10^{-3} / 1 \times 10^{-3}\end{array}$ & 99 & 1 & 15 & 50.8 & 107 & 1.51 \\
\hline 41 & & NFHMA & $\begin{array}{c}0.71 / 4 \times 10^{-4} \\
/ 1 \times 10^{-3} / 1 \times 10^{-3}\end{array}$ & 99 & 1 & 15 & 29.0 & 110 & 0.88 \\
\hline 42 & & HDFDA & $\begin{array}{c}0.52 / 4 \times 10^{-4} \\
/ 1 \times 10^{-3} / 1 \times 10^{-3}\end{array}$ & 99 & 1 & 15 & 52.4 & 119 & 0.86 \\
\hline 43 & $\begin{array}{l}\text { Glass } \\
\text { (small) }\end{array}$ & HDFDA & $\begin{array}{c}0.52 / 4 \times 10^{-4} \\
/ 1 \times 10^{-3} / 1 \times 10^{-3}\end{array}$ & 99 & 1 & 15 & NA & 114 & 1.73 \\
\hline 44 & $\begin{array}{l}\text { Glass } \\
\text { (large) }\end{array}$ & HDFDA & $\begin{array}{c}0.52 / 4 \times 10^{-4} \\
/ 1 \times 10^{-3} / 1 \times 10^{-3}\end{array}$ & 99 & 1 & 15 & NA & 117 & NA \\
\hline 45 & PC & HDFDA & $\begin{array}{c}0.52 / 4 \times 10^{-4} \\
/ 1 \times 10^{-3} / 1 \times 10^{-3}\end{array}$ & 99 & 1 & 15 & NA & 114 & 0.53 \\
\hline 46 & PET & HDFDA & $\begin{array}{c}0.52 / 4 \times 10^{-4} \\
/ 1 \times 10^{-3} / 1 \times 10^{-3}\end{array}$ & 99 & 1 & 30 & NA & 113 & 0.40 \\
\hline
\end{tabular}



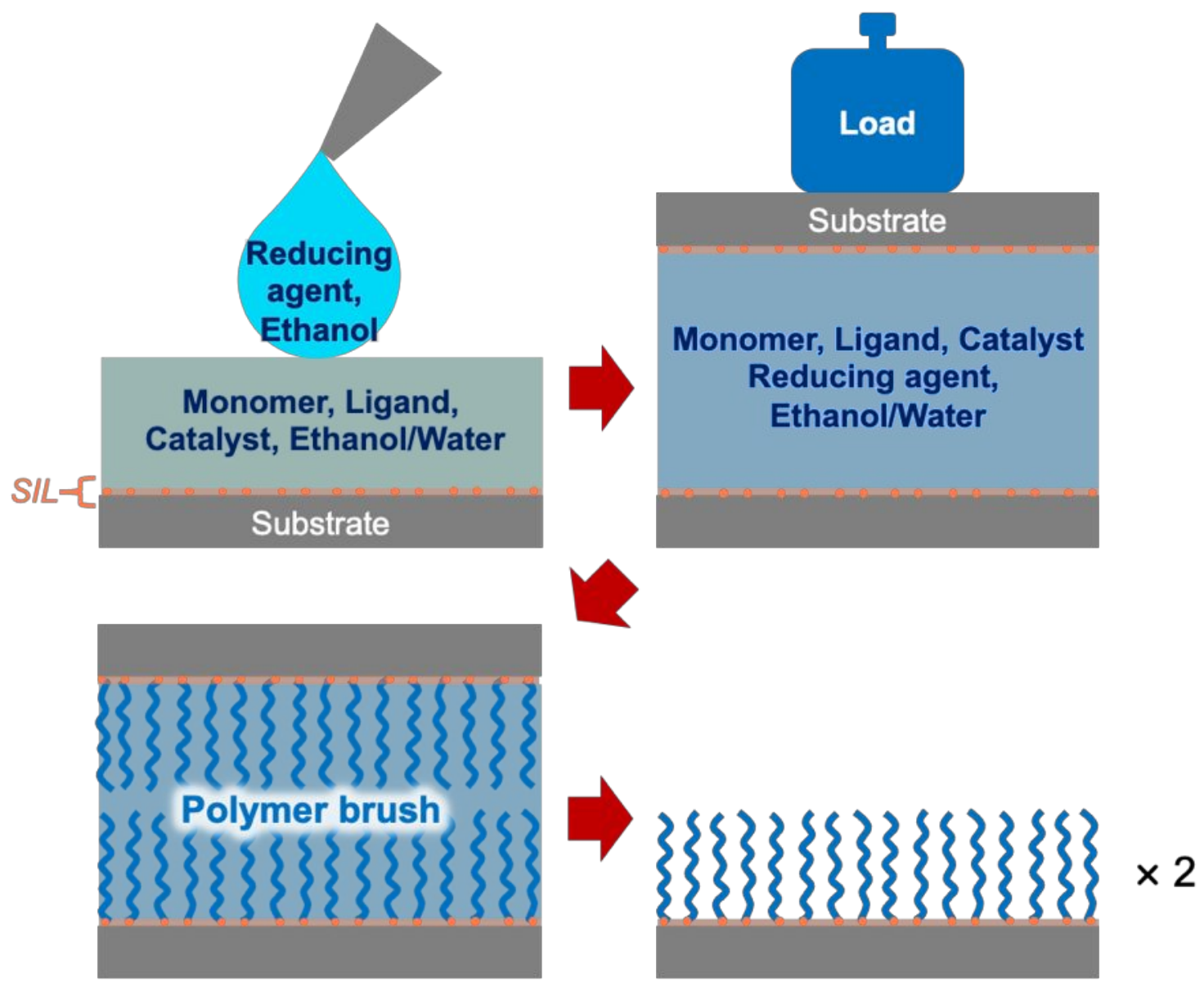

Figure S1. Schematic illustration of setup for water-accelerated "paint-on" SI-ATRP demonstrated on SIL-grafted large-scale substrates.

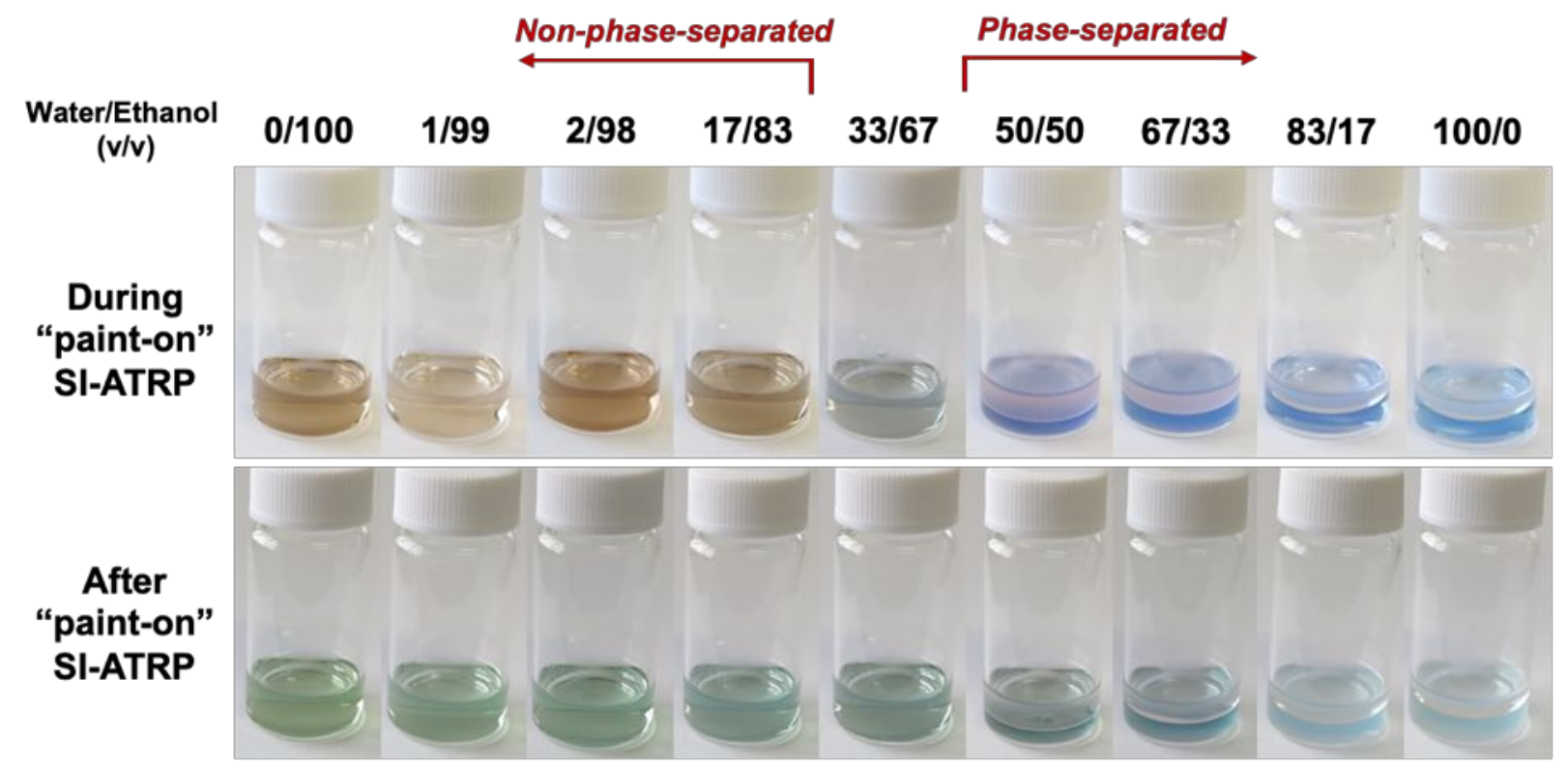

Figure S2. Color changes of reaction solutions for water-accelerated "paint-on" SI-ATRP of methyl methacrylate (MMA) during ( $\sim \mathrm{min}$, after activation) and after (completely deactivated) the reaction. 

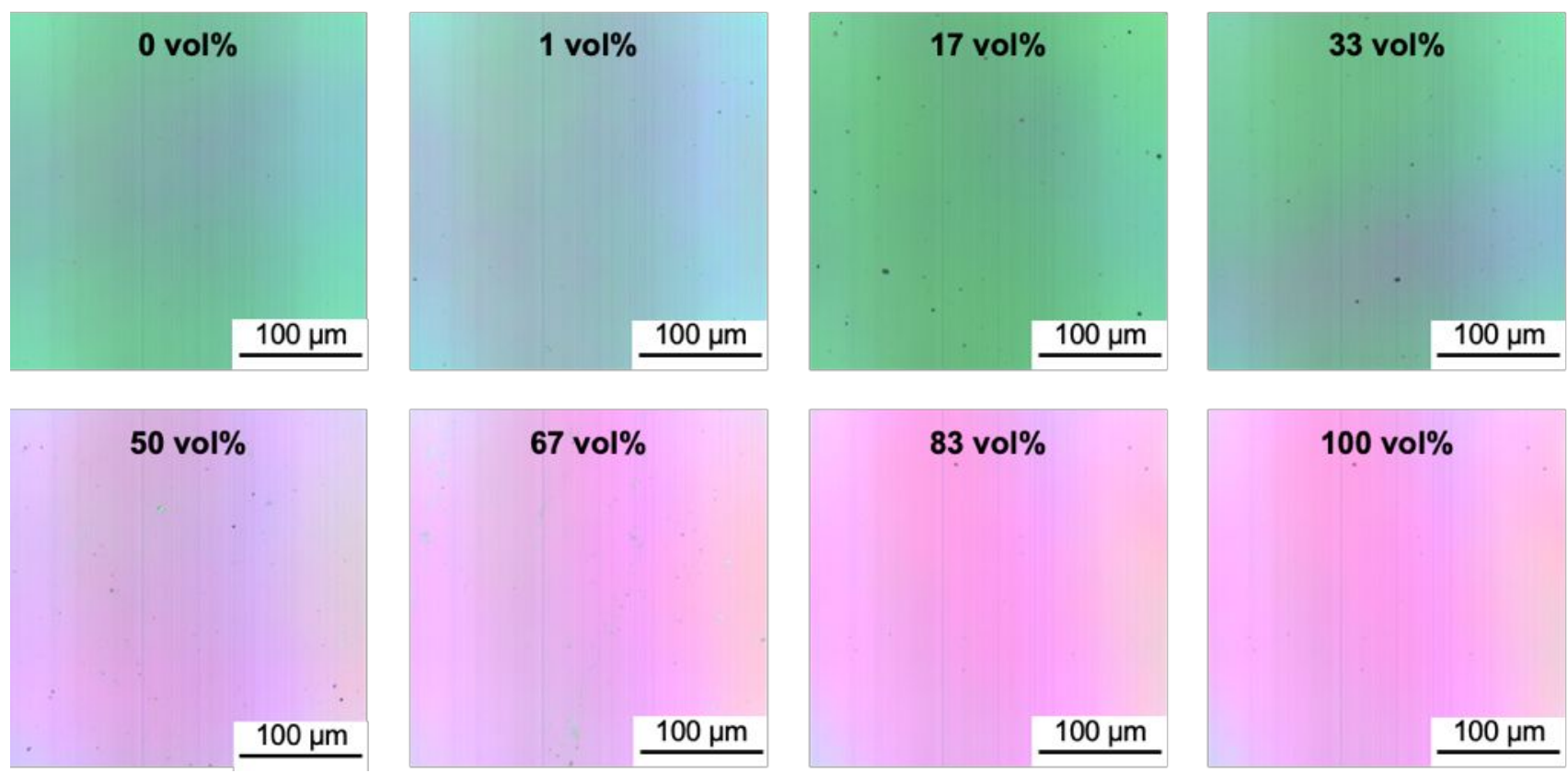

Figure S3. Typical optical microscope (OM, viewing area of $\left.300 \times 300 \mu \mathrm{m}^{2}\right)$ images of PMMA brush-grafted Si substrates prepared by water-accelerated "paint-on" SI-ATRP at different ethanol/water mixing ratio.
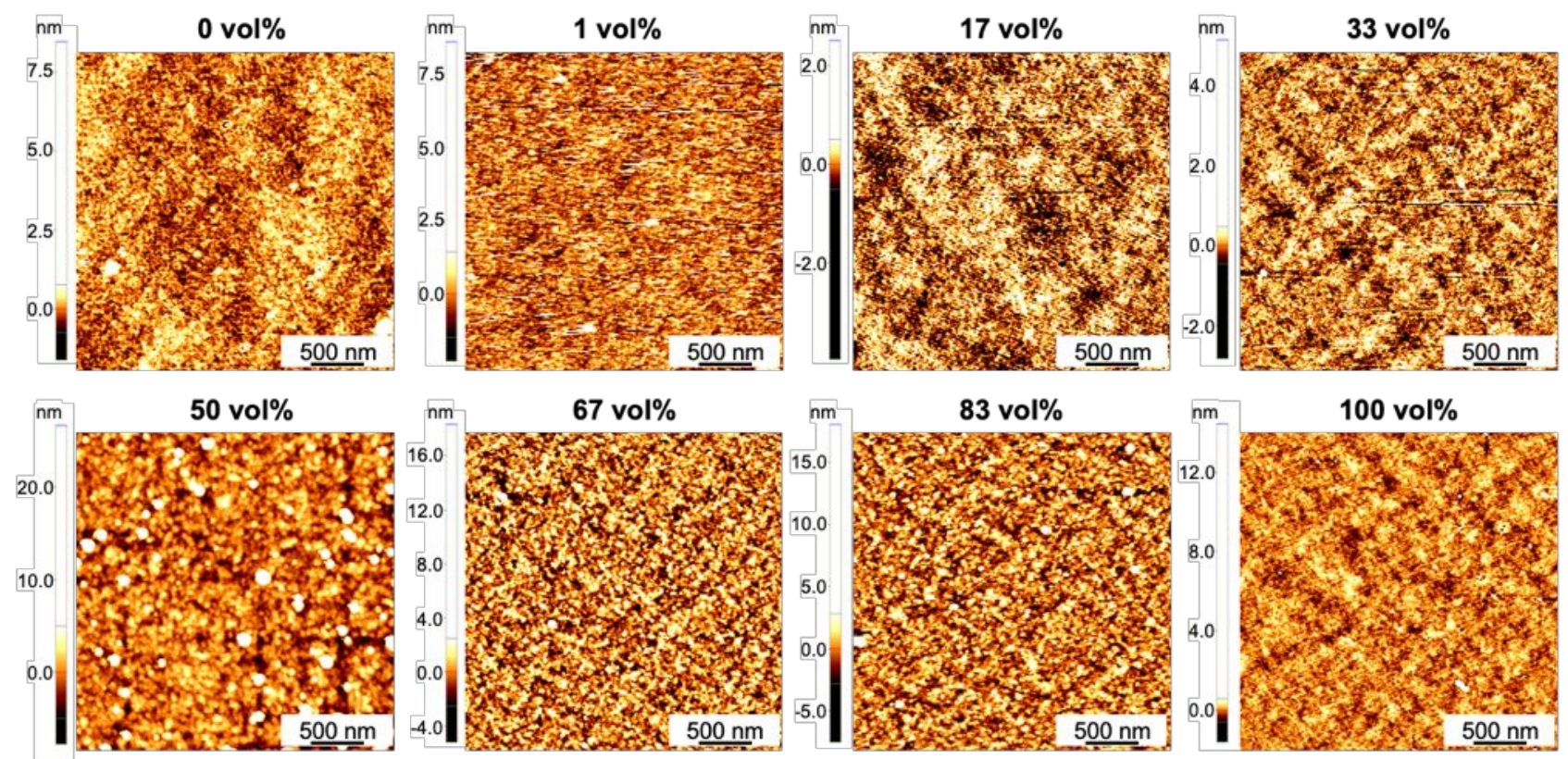

Figure S4. Typical atomic force microscope (AFM, viewing area of $\left.3 \times 3 \mu \mathrm{m}^{2}\right)$ images of PMMA brush-grafted Si substrates prepared by water-accelerated "paint-on" SI-ATRP at different ethanol/water mixing ratio. 


\section{Durability Test of "paint-on SI-ATRP Reaction Solution}

To evaluate stability and durability our "paint-on" systems, PMMA brushes was prepared on SIL-covered Si substrates through a water-accelerated "paint-on" SI-ATRP process after leaving the precursor solutions under the lab conditions for long periods of time $(24 \mathrm{~h}$ or $240 \mathrm{~h})$. First, three ethanolic solutions of MMA ( $2 \mathrm{~mL}$ in $1.5 \mathrm{~mL}$ ethanol/water $(98 / 2 \mathrm{v} / \mathrm{v})$ mixture), copper (II) chloride and $N, N, N, N^{\prime}, N^{\prime}$ 'pentamethyldiethylenetriamine ( $2.8 \mathrm{mg}$ and $10 \mu \mathrm{L}$ in $4 \mathrm{~mL}$ ethanol), and ascorbic acid (40 $\mathrm{mg}$ in $4 \mathrm{~mL}$, ultrasonicated for $5 \mathrm{~min}$ to achieve complete dissolution) were separately prepared. Then, SI-ATRP of MMA was carried out as follows:

A) all chemicals including monomer, water, catalyst, ligand, and reducing agent were added to a glass vial together with a piece of SIL-covered Si substrate $(1 \mathrm{~cm} \times 2 \mathrm{~cm})$. The solution was mixed, stored under the lab conditions for 24 or $240 \mathrm{~h}$, and then used for SI-ATRP (Process A).

B) The ethanolic solutions of monomer/water, catalyst/ligand, and reducing agent were separately stored under the lab conditions for 24 or $240 \mathrm{~h}$. Then, they were added to a glass vial together with a piece of SIL-covered Si substrate $(1 \mathrm{~cm} \times 2 \mathrm{~cm})$, mixed, and used for SI-ATRP (Process B).

Polymerizations were conducted at room temperature under ARGET conditions without stirring. After $15 \mathrm{~min}$, the samples were removed from the solution, and then extensively rinsed with toluene, ethanol and Mill-Q water repeatedly, and were blown dry with $\mathrm{N}_{2}$ gas. 
Table S4 Dry thicknesses, water CAs, and $R_{\text {rms }}$ values of the resulting PMMA brushes grafted from SIL-formed Si substrates by water-accelerated "paint-on" SI-ATRP using stored solutions for 24 or $240 \mathrm{~h}$

\begin{tabular}{|c|c|c|c|c|c|c|c|c|c|}
\hline Entry & Process & $\begin{array}{l}\text { Storage } \\
\text { time } \\
\text { (hours) }\end{array}$ & Substrate & Monomer & $\begin{array}{l}{[\text { monomer }] /\left[\mathrm{CuCl}_{2}\right]} \\
/[\mathrm{PMDETA}] /[\mathrm{AA}]\end{array}$ & $\begin{array}{l}\text { Ethanol } \\
\text { (vol\% in } \\
\text { solvent) }\end{array}$ & $\begin{array}{l}\text { Water } \\
\text { (vol\% in } \\
\text { solvent) }\end{array}$ & $\begin{array}{l}\text { Reaction } \\
\text { time } \\
\text { (min) }\end{array}$ & $\begin{array}{l}\text { Thickness } \\
\text { (nm) }\end{array}$ \\
\hline 47 & . & 24 & \multirow{4}{*}{$\mathrm{Si}$} & \multirow{4}{*}{ MMA } & \multirow{4}{*}{$\begin{array}{c}1.5 / 4 \times 10^{-4} \\
/ 1 \times 10^{-3} / 1 \times 10^{-3}\end{array}$} & \multirow{4}{*}{98} & \multirow{4}{*}{2} & \multirow{4}{*}{15} & 0.02 \\
\hline 48 & $\mathrm{~A}$ & 240 & & & & & & & $<0.01$ \\
\hline 49 & \multirow{2}{*}{ B } & 24 & & & & & & & 41.1 \\
\hline 50 & & 240 & & & & & & & 40.9 \\
\hline
\end{tabular}

\section{Influence of Monomer Concentration on "paint-on" SI-ATRP Kinetics}

To investigate the influence of monomer concentration on the kinetics of our "painton" SI-ATRP process, PMMA brushes were grafted by varying monomer concentrations. Briefly, a proper amount of MMA (concentrations were set at 0.1-50 vol\%, Table S4), $\mathrm{CuCl}_{2}$, PMEDTA, water, and ethanol were added to a glass vial with a piece of SIL-formed Si substrate $(2 \mathrm{~cm} \times 1 \mathrm{~cm})$, and then mixed. Freshly prepared ethanolic solution of AA $(10 \mathrm{mg} / \mathrm{mL})$ was then added to the reaction solutions and stirred for about $5 \mathrm{sec}$ to activate the catalyst. Polymerizations were allowed to proceed at room temperature under ARGET conditions without stirring. After $15 \mathrm{~min}$, the substrates were removed from the solution. They were then extensively rinsed with toluene, ethanol and Mill-Q water repeatedly to remove excess monomer, and were dried with a $\mathrm{N}_{2}$ blowing at room temperature. 
Table S5 Dry thicknesses of the resulting PMMA brushes grafted from SIL-formed Si substrates by water-accelerated "paint-on" SI-ATRP at various monomer concentrations

\begin{tabular}{|c|c|c|c|c|c|c|}
\hline Entry & Substrate & Monomer & $\begin{array}{l}\text { Ethanol } \\
\text { (vol\% in } \\
\text { solvent) }\end{array}$ & $\begin{array}{l}\text { Water } \\
\text { (vol\% in ( } \\
\text { solvent) }\end{array}$ & $\begin{array}{l}\text { Monomer } \\
\text { Concentration } \\
(\text { vol\%) }\end{array}$ & $\begin{array}{l}\text { Thickness } \\
\text { (nm) }\end{array}$ \\
\hline 51 & \multirow{5}{*}{$\mathrm{Si}$} & \multirow{5}{*}{ MMA } & \multirow{5}{*}{98} & \multirow{5}{*}{2} & 50 & 40.9 \\
\hline 52 & & & & & 25 & 26.2 \\
\hline 53 & & & & & 10 & 1.3 \\
\hline 54 & & & & & 1 & $<0.1$ \\
\hline 55 & & & & & 0.1 & $<0.1$ \\
\hline
\end{tabular}

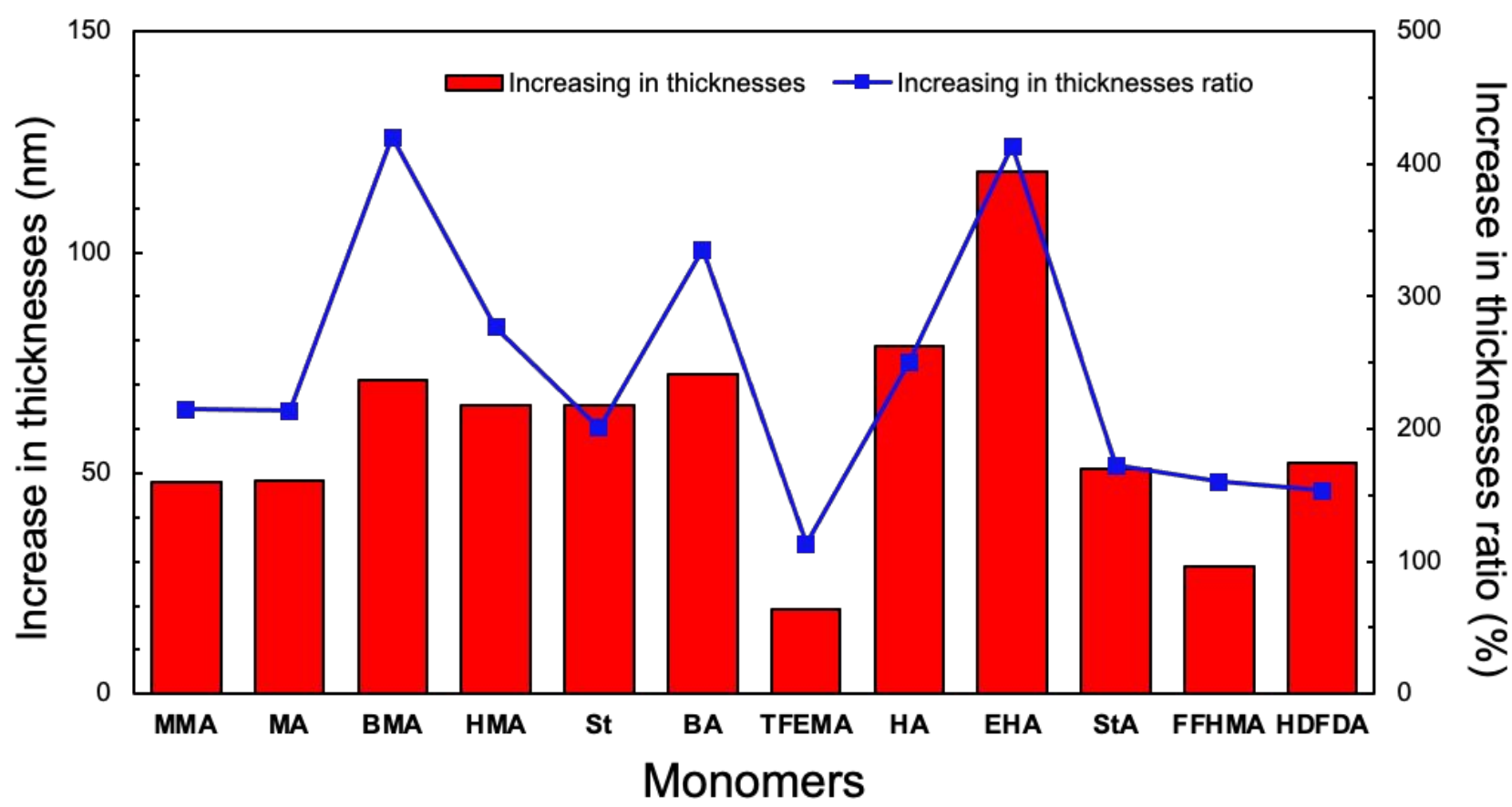

Figure S5. Increases in thicknesses and thicknesses ratio of a wide variety of hydrophobic polymer brushes prepared by water-accelerated "paint-on" SI-ATRP comparing to "paint-on" SI-ATRP without addition of water. 


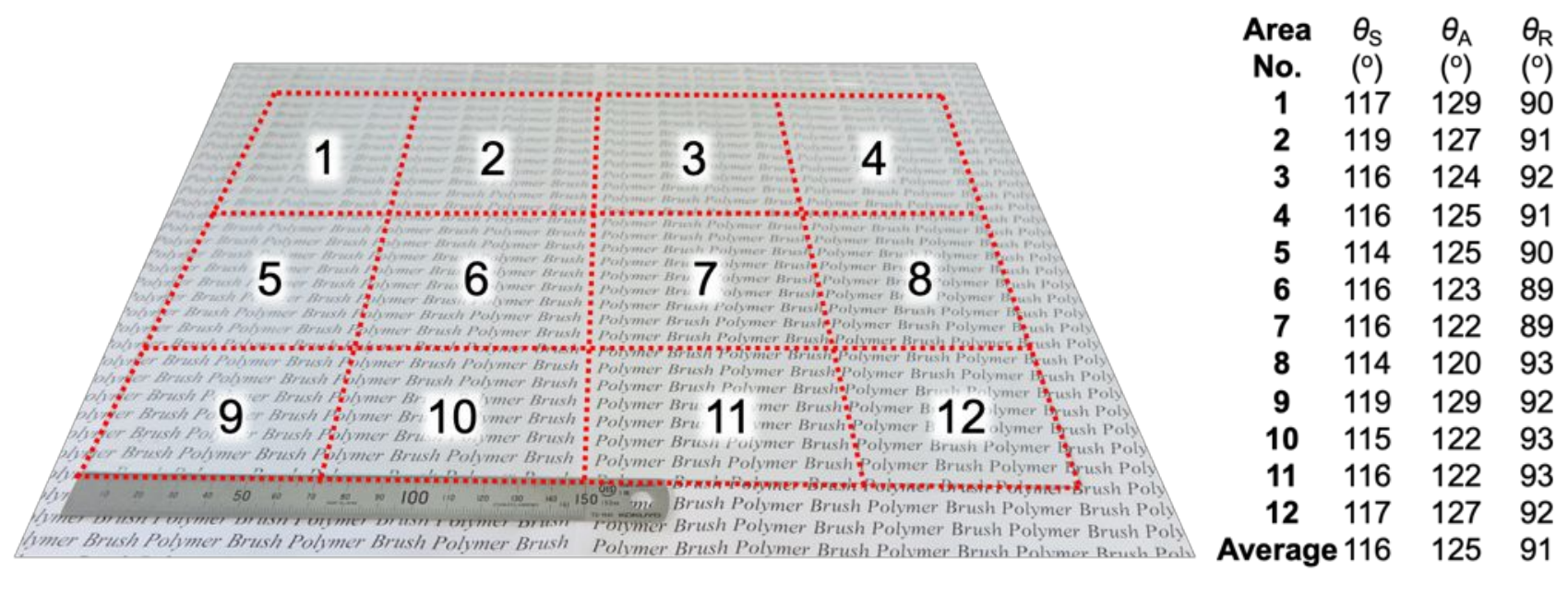

Figure S6. Appearance and distributions of water CAs of the poly $(1 \mathrm{H}, 1 \mathrm{H}, 2 \mathrm{H}, 2 \mathrm{H}$ heptadecafluorodecyl acrylate) (PHDFDA) brush layer formed on large-scale glass substrate. 
Table S6 Static and dynamic CAs of five probe liquids on PHDFDA brush-grafted Si substrates ( $\theta_{\mathrm{A}}$ : advancing $\mathrm{CA}, \theta_{\mathrm{R}}$ : receding $\mathrm{CA}, \Delta \theta$ : CA hysteresis, and $\theta_{\mathrm{T}}$ : sliding/tilt angle)

\begin{tabular}{|c|c|c|c|c|c|c|c|}
\hline Probe liquid & $\begin{array}{l}\text { Surface tension } \\
\quad(\mathrm{mN} / \mathrm{m})\end{array}$ & Substrate & $\begin{array}{l}\theta_{\mathrm{S}} \\
\left(^{\circ}\right)\end{array}$ & $\begin{array}{l}\theta_{\mathrm{A}} \\
\left(^{\circ}\right)\end{array}$ & $\begin{array}{l}\theta_{\mathrm{R}} \\
\left(^{\circ}\right)\end{array}$ & $\begin{array}{l}\Delta \theta \\
\left(^{\circ}\right)\end{array}$ & $\begin{array}{l}\theta_{\mathrm{T}} \\
\left({ }^{\circ}\right)\end{array}$ \\
\hline \multirow{5}{*}{ Water } & \multirow{5}{*}{72.8} & $\mathrm{Si}$ & 114 & 116 & 96 & 20 & 16 \\
\hline & & Glass (small) & 118 & 127 & 97 & 30 & 15 \\
\hline & & Glass (large) & 117 & 127 & 94 & 33 & 18 \\
\hline & & $\mathrm{PC}$ & 114 & 118 & 93 & 25 & 19 \\
\hline & & PET & 113 & 119 & 87 & 32 & 21 \\
\hline \multirow{5}{*}{ Diiodomethane } & \multirow{5}{*}{50.8} & $\mathrm{Si}$ & 103 & 106 & 88 & 18 & 9 \\
\hline & & Glass (small) & 103 & 107 & 93 & 14 & 8 \\
\hline & & Glass (large) & 100 & 109 & 90 & 19 & 14 \\
\hline & & $\mathrm{PC}$ & 109 & 115 & 89 & 26 & 13 \\
\hline & & PET & 104 & 109 & 89 & 20 & 13 \\
\hline \multirow{5}{*}{ Ethylene glycol } & \multirow{5}{*}{46.5} & $\mathrm{Si}$ & 104 & 110 & 97 & 13 & 15 \\
\hline & & Glass (small) & 105 & 108 & 95 & 13 & 15 \\
\hline & & Glass (large) & 110 & 116 & 91 & 25 & 19 \\
\hline & & $\mathrm{PC}$ & 114 & 119 & 92 & 27 & 19 \\
\hline & & PET & 105 & 108 & 78 & 30 & 19 \\
\hline \multirow{5}{*}{$n$-Hexadecane } & \multirow{5}{*}{27.5} & $\mathrm{Si}$ & 81 & 84 & 73 & 11 & 12 \\
\hline & & Glass (small) & 83 & 84 & 70 & 14 & 11 \\
\hline & & Glass (large) & 82 & 85 & 69 & 16 & 15 \\
\hline & & $\mathrm{PC}$ & 86 & 89 & 69 & 20 & 15 \\
\hline & & PET & 78 & 82 & 50 & 32 & 15 \\
\hline
\end{tabular}


Movie S1. Motion of water droplet placed on the inclined PHDFDA brush-grafted glass slide $(5 \mathrm{~cm} \times$ $8 \mathrm{~cm})$.

Movie S2. Motion of $n$-hexane droplet placed on the inclined PHDFDA brush-grafted glass slide (5 $\mathrm{cm} \times 8 \mathrm{~cm})$.

Movie S3. Continuous water jet flow on PHDFDA brush-grafted glass slide $(5 \mathrm{~cm} \times 8 \mathrm{~cm})$.

Movie S4. Continuous oil ( $n$-hexane) jet flow on PHDFDA brush-grafted glass slide $(5 \mathrm{~cm} \times 8 \mathrm{~cm})$.

\section{References}

1. Yamaguchi, H.; Kikuchi, M.; Kobayashi, M.; Ogawa, H.; Masunaga, H.; Sakata, O.; Takahara, A. Influence of Molecular Weight Dispersity of Poly\{2-(Perfluorooctyl)Ethyl Acrylate $\}$ Brushes on Their Molecular Aggregation States and Wetting Behavior. Macromolecules 2012, 45, $1509-1516$.

2. Patil, R. R.; Turgman-Cohen, S.; Šrogl, J.; Kiserow, D.; Genzer, J. On-Demand Degrafting and the Study of Molecular Weight and Grafting Density of Poly(methyl methacrylate) Brushes on Flat Silica Substrates. Langmuir 2015, 31, 2372-2381. 